\title{
Hypertrophie gingivale révélant une leucémie myélomonocytaire aiguë
}

\section{Gingival Enlargement Revealing Acute Myelomonocytic Leukemia}

\section{Leopold-Metzger $\cdot$ O. Peyrony $\cdot$ J.-P. Fontaine}

Reçu le 2 mai 2014; accepté le 18 juillet 2014

(C) SFMU et Springer-Verlag France 2014

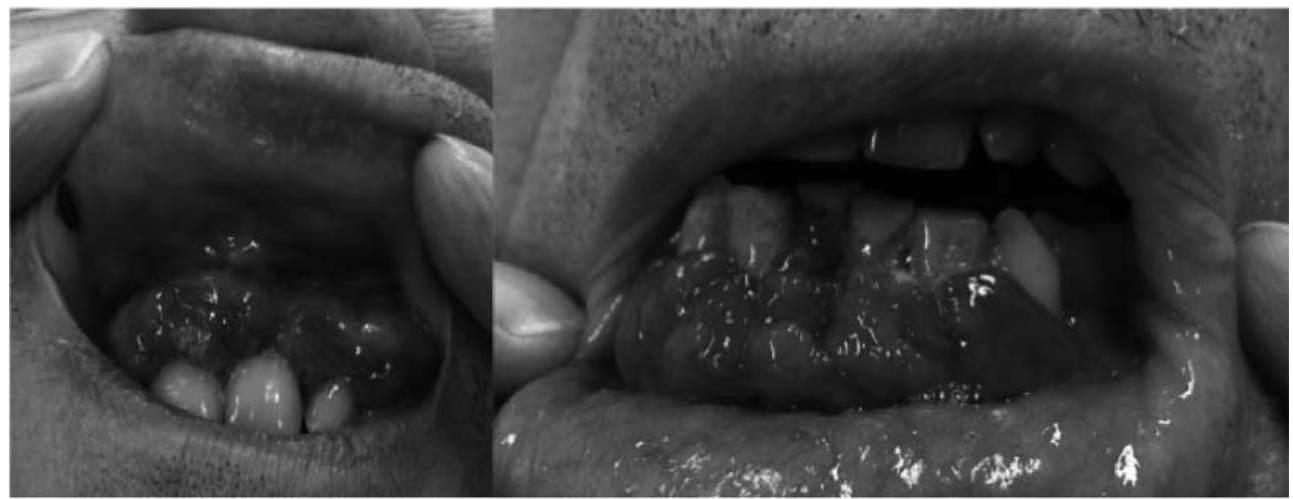

Fig. 1 Hypertrophie gingivale (muqueuse gingivale supérieure et inférieure hypertrophiée et inflammatoire)

Un homme de 74 ans aux antécédents d'asplénie congénitale et suivi pour une leucémie myélomonocytaire chronique (LMMC) consulte aux urgences pour une altération de l'état général avec difficultés à s'alimenter évoluant depuis deux mois. L'examen clinique objective une hypertrophie gingivale responsable de la dysphagie (Fig. 1). Il existe une hépatomégalie à deux travers de doigts sans adénomégalie. Le reste de l'examen est sans particularité. La biologie montre une majoration de l'hyperleucocytose $\left(299000 / \mathrm{mm}^{3}\right)$, une aggravation des cytopénies (hémoglobine à $7,9 \mathrm{~g} / \mathrm{dl}$ et 74000 plaquettes $\left./ \mathrm{mm}^{3}\right)$, l'apparition de blastes (11\%), des stigmates de lyse tumorale spontanée (élévation des LDH à $6460 \mathrm{U} / \mathrm{L}$ et hyperphosphorémie à $1,55 \mathrm{mmol} / \mathrm{L}$, les autres marqueurs de lyse étant négatifs : absence d'hyperkaliémie ou d'hyperuricémie) et une insuffisance rénale aiguë (créa-

D. Leopold-Metzger $(\bowtie)$

Université Paris VI-Pierre et Marie Curie, UFR de médecine,

site Saint-Antoine, 27, rue Chaligny, F-75571 Paris, France

e-mail : d.leopold.m@gmail.com

\section{O. Peyrony $\cdot$ J.-P. Fontaine}

Service des urgences, hôpital Saint-Louis,

Assistance publique-hôpitaux de Paris,

1, avenue Claude-Vellefaux, F-75010 Paris, France tininémie à $263 \mu \mathrm{mol} / \mathrm{L}$ ). Le myélogramme réalisé aux urgences est en faveur d'une acutisation de la LMMC. Un traitement par hydroxyurée est initié en hématologie mais l'état du patient se dégrade rapidement dans un tableau associant une majoration du syndrome de lyse et de l'insuffisance rénale, une coagulation intravasculaire disséminée et une détresse respiratoire sur leucostase pulmonaire imposant son transfert en réanimation le lendemain. Des anomalies gingivales peuvent survenir dans 3 à $5 \%$ des leucémies myéloïdes aiguës, notamment au cours des leucémies myélomonocytaires et monoblastiques (LAM 4 et LAM 5) [1]. Les autres causes d'hypertrophie gingivale sont la gingivite chronique, la grossesse, la puberté, la carence en vitamine C, certains médicaments (phénitoïne, inhibiteurs calciques, cyclosporine), certaines maladies systémiques (granulomatose de Wegener, sarcoïdose, maladie de Crohn, amylose, sarcome de Kaposi, neurofibromatose). La découverte d'une hypertrophie gingivale aux urgences doit faire éliminer une leucémie aiguë.

\section{Référence}

1. Khera P, Zirwas MJ, English JC (2005) Diffuse gingival enlargement. J Am Acad Dermatol 52:491-9 